\title{
Pore Network Simulations of Heat and Mass Transfer inside an Unsaturated Capillary Porous Wick in the Dry-out Regime
}

\author{
Kieu Hiep Le ${ }^{1}$. Abdolreza Kharaghani ${ }^{1}$. \\ Christoph Kirsch ${ }^{2}$. Evangelos Tsotsas ${ }^{1}$
}

Received: 7 August 2015 / Accepted: 25 June 2016 / Published online: 4 July 2016

C Springer Science+Business Media Dordrecht 2016

\begin{abstract}
In this work, a two-dimensional pore network model is developed to study the heat and mass transfer inside a capillary porous wick with opposite replenishment in the dry-out regime. The mass flow rate in each throat of the pore network is computed according to the Hagen-Poiseuille law, and the heat flux is calculated based on Fourier's law with an effective local thermal conductivity. By coupling the heat and the mass transfer, a numerical method is devised to determine the evolution of the liquid-vapor interface. The model is verified by comparing the effective heat transfer coefficient versus heat load with experimental observations. For increasing heat load, an inflation/deflation of the vapor pocket is observed. The influences of microstructural properties on the vapor pocket pattern and on the effective heat transfer coefficient are discussed: A porous wick with a non-uniform or bimodal pore size distribution results in a larger heat transfer coefficient compared to a porous wick with a uniform pore size distribution. The heat and mass transfer efficiency of a porous wick comprised of two connected regions of small and large pores is also examined. The simulation results indicate that the introduction of a coarse layer with a suitable thickness strongly enhances the heat transfer coefficient.
\end{abstract}

Keywords Pore network model · Heat and mass transfer · Vapor pocket - Inflation and deflation · Porous wick

\section{List of symbols}

$\begin{array}{ll}A & \text { Area }\left(\mathrm{m}^{2}\right) \\ e & \text { Relative coarse layer thickness }\end{array}$

Abdolreza Kharaghani

abdolreza.kharaghani@ovgu.de

1 Thermal Process Engineering, Otto von Guericke University, P.O. 4120, 39106 Magdeburg, Germany

2 Institute of Computational Physics, Zurich University of Applied Sciences, Wildbachstrasse 21, 8401 Winterthur, Switzerland 


$\begin{array}{ll}f_{\mathrm{v}} & \text { Area fraction of the vapor region } \\ \Delta H_{\text {evp }} & \text { Evaporation latent heat }(\mathrm{J} / \mathrm{kg}) \\ L & \text { Throat length }(\mathrm{m}) \\ \dot{M} & \text { Mass flow rate }(\mathrm{kg} / \mathrm{s}) \\ \boldsymbol{n} & \text { Outward unit normal vector } \\ p & \text { Pressure }(\mathrm{Pa}) \\ \dot{Q} & \text { Heat rate }(\mathrm{W}) \\ \dot{q} & \text { Heat load }\left(\mathrm{W} / \mathrm{m}^{2}\right) \\ r & \text { Throat radius }(\mathrm{m}) \\ r_{0} & \text { Mean throat radius }(\mathrm{m}) \\ T & \text { Temperature }\left({ }^{\circ} \mathrm{C}\right) \\ v & \text { Superficial velocity }(\mathrm{m} / \mathrm{s}) \\ v_{0} & \text { Interstitial velocity }(\mathrm{m} / \mathrm{s}) \\ W & \text { Network thickness }(\mathrm{m})\end{array}$

\section{Greek symbols}

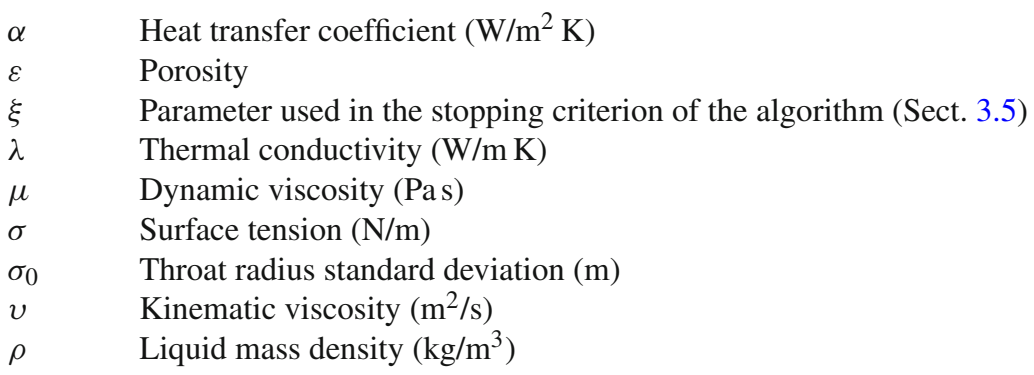

\section{Subscripts/superscripts}

$\begin{array}{ll}\mathrm{c} & \text { Capillary } \\ \mathrm{ch} & \text { Channel } \\ \mathrm{cv} & \text { Control volume } \\ \mathrm{eff} & \text { Effective } \\ \mathrm{evp} & \text { Evaporation } \\ \mathrm{f} & \text { Fluid } \\ \mathrm{fin} & \text { Fin } \\ i, j, k, m, n & \text { Pore } \\ \text { in } & \text { Inlet } \\ \text { inc } & \text { Incipient } \\ \mathrm{l} & \text { Liquid } \\ \text { out } & \text { Outlet } \\ \mathrm{s} & \text { Solid } \\ \text { sat } & \text { Saturation } \\ \mathrm{v} & \text { Vapor } \\ x, y & \text { Horizontal and vertical coordinates }\end{array}$




\section{Introduction}

Non-isothermal fluid flow with phase transition in porous media occurs in numerous heat exchange devices. The advantage of using porous material in the fabrication of these devices is its very large specific area, which enhances the heat and mass transfer process at the pore scale. The phase transition phenomena such as evaporation or condensation yield a very high value of the heat transfer coefficient, allowing devices to be more compact. Furthermore, the liquid phase can be transported through the porous medium by wicking, which is driven by the capillary suction taking place at the three-phase contact line. Therefore, the porous medium works like a capillary pump and thus moving parts are not needed in the equipment. Two major applications utilizing these benefits of two-phase flow porous wicks are the loop heat pipe (LHP) and the capillary pumped loop (CPL). The model considered here is inspired by a typical situation in LHP and CPL evaporators; however, this model is not limited to this context. Therefore, this article will not use the LHP terminology except in Sect. 4, where the pattern of the vapor pocket in an LHP is discussed.

A porous layer in which the liquid and the thermal energy are replenished at opposite sides is considered in this study. This configuration appears in several applications, not only in LHP evaporators (Faghri 1995), but also in porous burners (Jugjai and Phothiya 2007), for example. As shown in the two-dimensional cross-sectional image (Fig. 1), the liquid is supplied from the bottom and it invades the porous wick due to capillary action. The heat load is applied at the top surface via a metallic fin, which is in contact with the porous structure. For high heat load the liquid evaporates inside the wick, and the vapor flows toward the open segments of the top surface. The heat and mass transfer processes are thus strongly coupled in this device. Many parameters and properties may influence the heat and mass transfer inside the capillary porous wick, such as the solid phase material, thickness, porosity, and permeability of the porous wick, the working fluid, and the heat load as well as other operating conditions. Parametric studies shall allow us to select some of these parameters for evaluation and to analyze the behavior of the system under parameter variations.

Conventionally, as assumed in the literature (Figus et al. 1999; Siedel et al. 2015), the system can be in one of two different regimes: In the low heat load regime, the porous wick is fully wetted and the evaporation occurs only at the wick surface. In the high heat load regime, however, the porous wick is only partially saturated and evaporation occurs also inside the wick. Cao and Faghri (1994a) developed a two-dimensional analytical model for the heat and mass transfer in a fully wetted porous wick. In their model, the liquid and vapor flows are coupled via the mass balance equation at the porous wick surface. The liquid flow in the porous wick and the vapor transport between the fins are determined from numerical simulations using the finite element method. In a later study of the same authors (Cao and Faghri 1994b), an approximate analytical solution of the model equations is given for flat segments of the porous wick. Li and Peterson (2011) developed three-dimensional models for a square flat wick, which has a liquid reservoir at the top and a heating substrate at the bottom. Chernysheva and Maydanik (2012) as well as Zhang et al. (2012) presented 3D models for capillary porous wicks with longitudinal replenishment. In these studies, the liquid flow is assumed to be perpendicular to the heat flux at the wick wall, whereas in Li and Peterson (2011) the fluid and energy flows are counter current. The model in Chernysheva and Maydanik (2012) accounts for the dependence of the thermal properties on the temperature (but not on the pressure), whereas the other models mentioned so far assume constant thermal properties. Since these models are restricted to fully wet homogeneous and isotropic porous wicks, they are valid only in the low heat load regime. 
For high heat load (the so-called dry-out regime), several heat and mass transfer models for the unsaturated porous wick were developed. Zhao and Liao (2000) presented a 1D analytical model to determine the heat and mass transfer in the porous layer wick. They assumed that the working fluid is a mixture of liquid and vapor in the two-phase zone, and therefore, the capillary effect and the liquid-vapor interface are not considered in their model. A 2D model based on Darcy's law showing the vapor zone inside a flat wick was presented by Demidov and Yatsenko (1994). This mathematical model used the moving evaporation front method of Figus et al. (1999). The Demidov and Yatsenko model was also used by Kaya and Goldak (2006) to investigate the heat and mass transfer in a cylindrical wick. In these models, the incipient boiling superheat value, the operating point at which the fully saturated wick changes to a partially wet wick, is assumed to be constant, whereas in Kaya and Goldak (2006) the incipient boiling superheat value was defined and discussed based on bubble nucleation in liquids. A time-dependent 2D continuum model for an unsaturated wick was presented by Boubaker et al. (2015). In that study, the evaporation rate is described by the Langmuir law instead of using energy balance equations as in the rest of studies corresponding to the dry-out regime of porous wick. Ren et al. (2007) and Ren (2011) developed an axisymmetric 2D model to determine the shape and position of the vapor zone for different values of the heat load in a cylindrical porous wick. In their studies, the capillary forces are taken into account, but the pressure jump across the liquid-vapor interface induced by the capillary pressure thresholds is not present in the pressure profiles (Figus et al. 1999; Ren et al. 2007; Ren 2011). The previous work cited up to here considers homogeneous and isotropic porous wicks in which the liquid-vapor interface is smooth. Therefore, these models cannot explain the evolution of a non-smooth liquid-vapor interface, which was observed, however, in experimental studies by Zhao and Liao (Liao and Zhao 1999, 2000; Zhao and Liao 2000), and by Byon and Kim (2012). In addition to continuum approaches, discrete pore network models (PNMs) provide another way to investigate flow and transport in porous media. Prat and his co-workers (Figus et al. 1999; Prat 2010; Mottet et al. 2014) used this approach to simulate the heat and mass transfer in $2 \mathrm{D}$ and in $3 \mathrm{D}$ capillary wicks at steady state. They also investigated the transient behavior of the capillary porous wick by a $2 \mathrm{D}$ pore network simulation (Louriou and Prat 2012).

Very recently, Mottet et al. (2015) and Nishikawara et al. (2015) presented 3D pore network models for different fluid configurations. In Mottet et al. (2015), the thermally conductive fin is made of a solid metallic blade whereas in Nishikawara et al. (2015) an etched porous fin is considered. The 3D simulation results (Mottet et al. 2015) indicate that between the saturated wick and the dry-out regime there is another operating regime, the so-called two-phase zone regime, in which the liquid and the vapor phase coexist below the fin. In this two-phase zone regime, the bottom surface of the fin is partially wetted; therefore, the effective heat transfer coefficient stabilizes at a high value before decreasing significantly during the dry-out regime. This interesting finding obtained by numerical simulations still needs to be confirmed experimentally. The appearance of the two-phase zone regime leads to a qualitative analogy between the calculated and experimentally observed thermal conductances (Mottet et al. 2015). Due to the assumption of a macroscopically sharp evaporation front in the continuum approach this regime was overlooked in some previous studies based on both 2D pore network and 2D/3D continuum simulations (Mottet et al. 2015). In these studies the vapor pocket pattern is visualized only for pore networks with a unimodal pore size distribution and for constant thermal properties of the liquid and vapor phases.

No exhaustive research work currently exists on the heat and mass transfer processes inside a capillary porous wick with opposite replenishment or on the effects of operation and structural parameters on the heat transfer efficiency. A parametric study of the heat and 


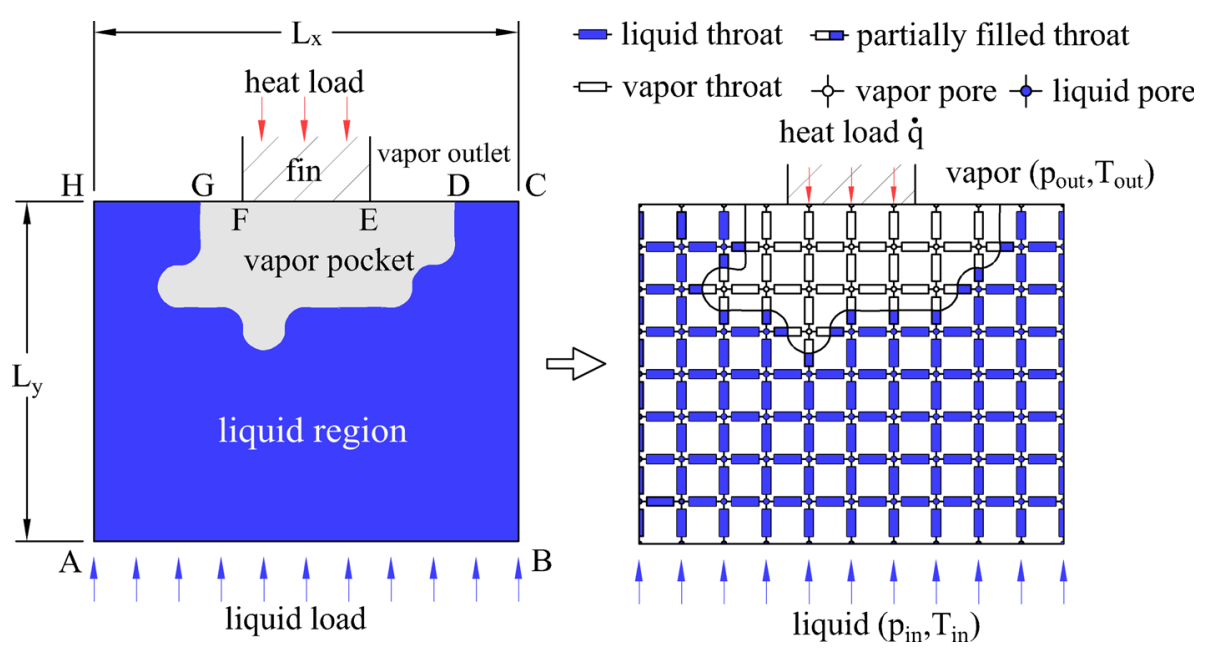

Fig. 1 Schematic 2D drawing of the heat and mass transfer processes in a porous wick (left), and their representation in a pore network model (right)

mass transfer in the porous wick is introduced in this paper. Although a 2D pore network model cannot represent the two-phase zone regime of a porous wick (Mottet et al. 2015), it still has the ability to predict the operating behavior in the dry-out regime for various porous structures. The energy and fluid transports are investigated by pore network models with discrete as well as continuous unimodal and bimodal pore size distributions. Bilayer porous wicks are also investigated.

\section{Problem Description}

A capillary wick with partial heating from the top surface is depicted in Fig. 1 (left). The liquid is supplied from the bottom of the wick, whereas the heat load is imposed near the top center of the wick. At the lateral edges of the wick symmetry boundary conditions are imposed. As discussed in the literature (Mottet et al. 2015; Siedel et al. 2015), for very high heat load regime, the capillary porous wick is in the dry-out regime. The incipient boiling superheat value is eventually reached as the heat load increases further, and a vapor zone, the so-called vapor pocket, forms inside the wick. Liquid is continuously pumped from the bottom to the liquid-vapor interface by capillary action. Evaporation occurs at the liquidvapor interface (both in the interior and at the surface of the porous wick), and the vapor flows toward the vapor outlet. A pore-scale representation of the unsaturated wick and of the processes involved is sketched in Fig. 1 (right). In this work, only steady-state operation is considered.

\section{Pore Network Model}

A porous wick is composed of a solid matrix and an interconnected network of closed and open pores (i.e., transport pores) filled with fluid (liquid or gas). The heat and mass transfer is considered to take place only in the open pores. As in several previous studies (Figus et al. 


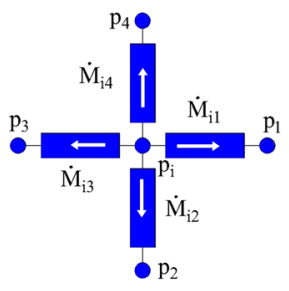

(a)

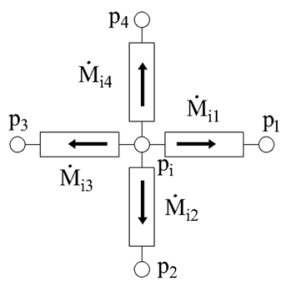

(b)

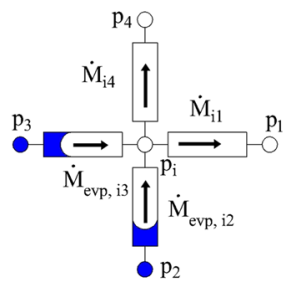

(c)

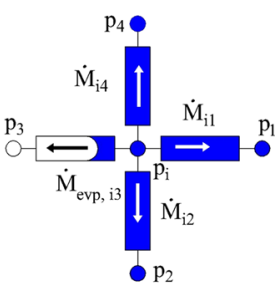

(d)

Fig. 2 Illustration of the mass balance for: $\mathbf{a}$ a liquid pore and $\mathbf{b}$ a vapor pore with empty neighboring throats, c vapor and $\mathbf{d}$ liquid pores with partially filled neighboring throats. $p$ denotes the pressure at the pores. Arrows indicate the flow directions of liquid or vapor

1999; Surasani et al. 2008; Louriou and Prat 2012), a 2D Cartesian network of pores (which have no volume) and cylindrical throats is employed to represent the microstructure of the porous wick (see Fig. 1). Each interior pore in the network is connected to four throats. The following assumptions were made in the development of our pore network model: The solid material is non-hygroscopic and saturated initially with a working fluid. Liquid films in the throats are neglected. The solid and fluid phases are in local thermal equilibrium. The liquid and vapor flows through the throats are laminar. Gravitational effects are assumed to be small compared to capillary effects, and thus neglected. Heat transfer is by conduction only. Despite these simplifications, the model is still capable of capturing striking features involved during the steady-state operation of an unsaturated capillary porous wick and provides insights into various macroscopic phenomena resulting from pore-scale processes. In the following, the pore network model is described in detail.

\subsection{Mass Transfer Model}

In this work, we assume that there is no non-condensate gas inside the wick. Hence, the Hagen-Poiseuille law is applied to compute the mass flow rate for both the liquid and the vapor phases:

$$
\dot{M}_{i j}=\frac{\pi r_{i j}^{4}}{8 v_{\mathrm{f}} L_{i j}}\left(p_{i}-p_{j}\right)
$$

where $\dot{M}_{i j}$ denotes the flow rate through the throat connecting the pores $i$ and $j, v_{\mathrm{f}}$ denotes the fluid (liquid/vapor) kinematic viscosity, $p_{i}$ and $p_{j}$ denote the liquid/vapor pressure at pores $i$ and $j$, respectively. $L_{i j}$ and $r_{i j}$ denote the throat length and radius, respectively.

To satisfy the mass conservation, the total mass flow at a pore $i$ which is located either inside the liquid region or inside the vapor pocket (see Fig. 2a, b) must be equal to zero:

$$
\sum_{j} \dot{M}_{i j}=0
$$

If, however, the pore $i$ connects to at least one partially liquid-filled throat (Fig. 2c, d), the mass conservation equation is instead given by

$$
\sum_{j} \dot{M}_{i j} \pm \sum_{k} \dot{M}_{\mathrm{evp}, i k}=0
$$

where the "+" sign is used in the case of a liquid pore and the "-" sign is used in the case of a vapor pore. In Eqs. 2 and 3, the sums run over all neighboring pores of pore $i ; j$ are the indices 


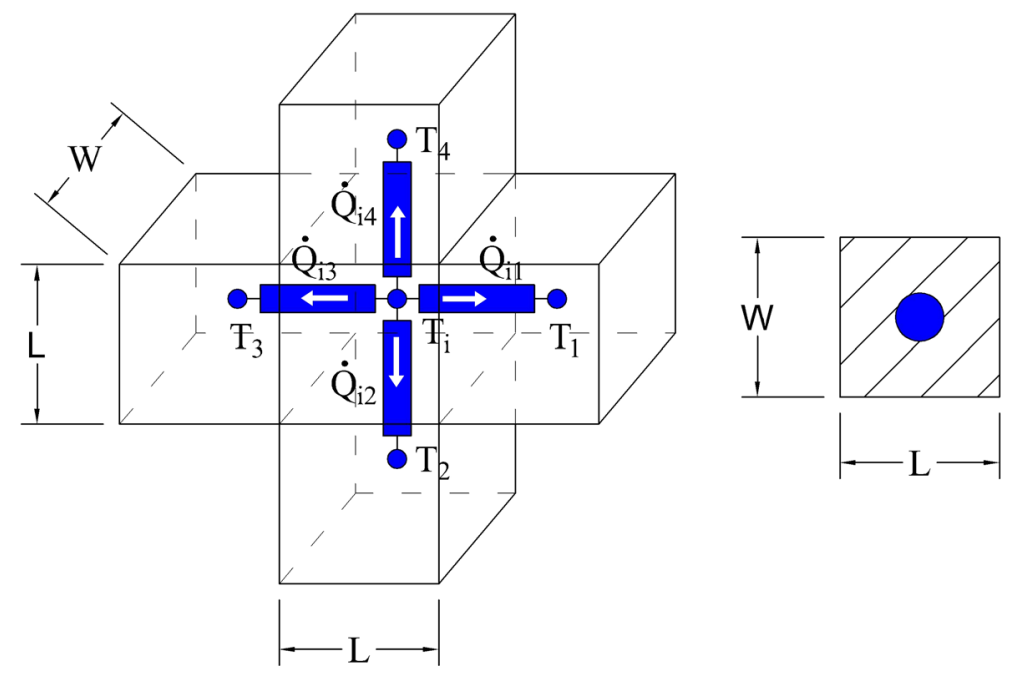

Fig. 3 Energy balance at a liquid pore $i$. Arrows inside the throats indicate the heat flow directions

of neighboring pores containing the same phase as pore $i$, and $k$ are the indices of neighboring pores containing a different phase than pore $i$. The evaporation flow rates, $\dot{M}_{\text {evp, } i k}$, are calculated by Eqs. 17 and 19. The resulting sparse system of linear equations (Eqs. 2, 3) is solved numerically to obtain the liquid and vapor pressure fields in the pore network.

\subsection{Heat Transfer Model}

Based on the local thermal equilibrium assumption, the temperatures of the solid and fluid phases must be equal. The heat transfer occurs only due to conduction in each phase. The evaporation latent heat at the liquid-vapor interface is taken into account in this model. The heat conduction in the vapor pocket or in the saturated wick is described by a discrete version of Fourier's law:

$$
\dot{Q}_{i j}=A_{\mathrm{cv}, i j} \lambda_{\mathrm{eff}, i j} \frac{T_{i}-T_{j}}{L}
$$

where $A_{\mathrm{cv}, i j}$ denotes the heat transfer area and where $\lambda_{\mathrm{eff}, i j}$ denotes the effective thermal conductivity between the pores $i$ and $j$. In a 2D Cartesian pore network, the heat transfer area is calculated as $A_{\mathrm{cv}, i j}=W L$ (see Fig. 3). Several models for the effective thermal conductivity $\lambda_{\text {eff }}$ can be found in the literature. It is usually a function of the solid phase conductivity, $\lambda_{s}$, the working fluid conductivity (vapor/liquid), $\lambda_{\mathrm{f}}$, and the ratio between the solid and void areas of connected throats. For the pore network model considered here, the effective thermal conductivity is determined by the parallel heat flux model (Tsotsas and Martin 1987):

$$
A_{\mathrm{cv}, i j} \lambda_{\mathrm{eff}, i j}=\left(A_{\mathrm{cv}, i j}-\pi r_{i j}^{2}\right) \lambda_{s}+\pi r_{i j}^{2} \lambda_{\mathrm{f}}
$$

For a pore $i$ in the vapor pocket or in the liquid cluster, the energy balance equation is given by

$$
\sum_{j} \dot{Q}_{i j}=\sum_{j} A_{\mathrm{cv}, i j} \lambda_{\mathrm{eff}, i j} \frac{T_{i}-T_{j}}{L}=0
$$

where $j$ runs over all pores connected to pore $i$. 


\subsection{Heat and Mass Transfer at the Liquid-Vapor Interface and at the Boundaries}

At the liquid-vapor interface, the evaporation is driven by heat transfer. The boundary conditions at the evaporation front in this work are as follows

$$
\begin{aligned}
& p_{\mathrm{v}}-p_{1}=p_{\mathrm{c}} \\
& T_{\mathrm{v}}=T_{1}=T_{\mathrm{sat}}\left(p_{\mathrm{v}}\right) \\
& \lambda_{\mathrm{eff}, v} \nabla T_{\mathrm{v}} \cdot \boldsymbol{n}-\lambda_{\mathrm{eff}, l} \nabla T_{1} \cdot \boldsymbol{n}=\rho_{1} v_{1} \cdot \boldsymbol{n} \cdot \Delta H_{\mathrm{evp}}
\end{aligned}
$$

In Eq. (7) $p_{\mathrm{v}}$ and $p_{1}$ denote the vapor and liquid pressure, respectively, and $p_{\mathrm{c}}=\frac{2 \sigma \cos \theta}{r}$ denotes the capillary pressure. In Eq. (8) $T_{\mathrm{v}}$ and $T_{1}$ denote the vapor and liquid temperature, respectively; $T_{\text {sat }}$ denotes the saturation temperature. In Eq. (9) $\lambda_{\text {eff, } v}$ and $\lambda_{\text {eff, } l}$ denote the effective thermal conductivity of vapor and liquid, respectively; $\boldsymbol{n}$ denotes the outward unit normal vector pointing out of the liquid phase; $\rho_{1}$ and $v_{1}$ denote the liquid mass density and velocity, respectively; $\Delta H_{\text {evp }}$ denotes the latent heat of the evaporation. The other boundary conditions are listed as follows [refer to Fig. 1 (left) for edge labels]:

At the bottom of the wick (edge $A B$ )

$$
T=T_{\text {in }}, \quad p=p_{\text {in }}
$$

where $T_{\text {in }}$ and $p_{\text {in }}$ denote the prescribed inlet liquid temperature and pressure, respectively. At the symmetric boundary (edges $A H$ and $B C$ )

$$
\frac{\partial T}{\partial x}=0, \quad \frac{\partial p}{\partial x}=0
$$

At the wick-fin interface (edge $E F$ )

$$
\lambda_{\text {eff }, v} \frac{\partial T}{\partial y}=\dot{q}, \quad \frac{\partial p}{\partial y}=0
$$

At the dry part of the vapor outlet (edges $D E$ and $F G$ )

$$
T=T_{\text {out }}, \quad p=p_{\text {out }}
$$

where $T_{\text {out }}$ and $p_{\text {out }}$ denote the prescribed vapor temperature and pressure at the vapor outlet, respectively. At the wet part of the vapor outlet (edges $C D$ and $G H$ )

$$
T=T_{\text {out }}, \quad-\lambda_{\text {eff }, l} \nabla T_{1} \cdot \boldsymbol{n}=\rho_{1} v_{1} \cdot \boldsymbol{n} \cdot \Delta H_{\text {evp }}
$$

These boundary conditions can be imposed directly in the pore network model, except the heat and mass transfer equations at the liquid-vapor interface (Eqs. 7-9 and 14). The evaporation rates $m_{\mathrm{evp}}=\rho_{1} v_{1} \cdot \boldsymbol{n}$ required at this interface are computed from energy balance equations (Eqs. 17, 19). Examples of a vapor pore and of a liquid pore at the liquid-vapor interface inside the wick, as well as of a liquid pore at the wet wick surface, are shown in Fig. 4. The interface conditions for these cases are explained in the following.

In the case where the front lies inside the wick (Fig. 4a), the temperatures at the liquid and vapor pores connected by a partially filled throat are given by the saturated temperature at the vapor pores, which depends on the vapor pressure,

$$
T_{i}=T_{k}=T_{\text {sat }}\left(p_{i}\right)
$$

The heat transfer condition (Eq. 9) at the liquid-vapor interface can be approximated by

$$
A_{\mathrm{cv}, m i} \lambda_{\mathrm{eff}, m i} \frac{T_{m}-T_{i}}{L}-A_{\mathrm{cv}, k n} \lambda_{\mathrm{eff}, k n} \frac{T_{k}-T_{n}}{L}=\Delta H_{\mathrm{evp}} \dot{M}_{\mathrm{evp}, i k}
$$




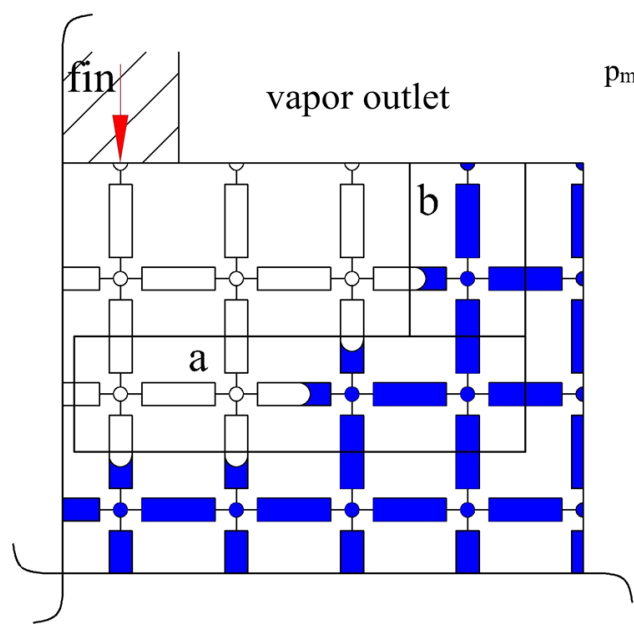

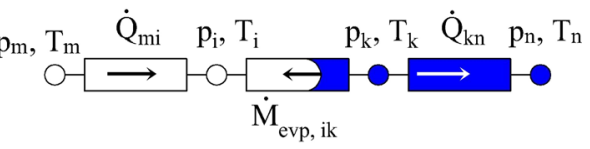

(a)

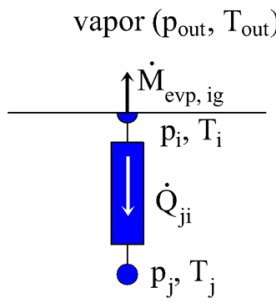

(b)

Fig. 4 Illustration of heat and mass transfer processes at the liquid-vapor interface, a inside the porous wick and $\mathbf{b}$ at the wick surface. Arrows indicate either mass or energy flow directions. The liquid and vapor phases are shown in blue and white, respectively

which can be solved for the evaporation rate:

$$
\dot{M}_{\mathrm{evp}, i k}=\frac{1}{\Delta H_{\mathrm{evp}}}\left(A_{\mathrm{cv}, m i} \lambda_{\mathrm{eff}, m i} \frac{T_{m}-T_{i}}{L}-A_{\mathrm{cv}, k n} \lambda_{\mathrm{eff}, k n} \frac{T_{k}-T_{n}}{L}\right)
$$

The pressure difference between the vapor pore and the liquid pore is given by the capillary pressure

$$
p_{i}-p_{j}=p_{c, i j}=\frac{2 \sigma \cos \theta}{r_{i j}}
$$

In the case where the liquid pore is located at the top surface of the wick (Fig. 4b), the evaporation rate from pore $i$ to the vapor outlet is computed by the heat balance equation

$$
\dot{M}_{\mathrm{evp}, i g}=\frac{A_{\mathrm{cv}, i j} \lambda_{\mathrm{eff}, i j}}{\Delta H_{\mathrm{evp}}} \frac{T_{j}-T_{i}}{L}
$$

\subsection{Working Fluid}

The working fluid in LHPs and CPLs is chosen based on the operating temperature (Faghri 1995). For a low operating temperature, ammonia or organic substances such as methanol or acetone are chosen as working fluid due to their low melting point and high vapor density. In the very high operating temperature range, the heat pipe is filled by liquid metal such as sodium or potassium. In this study, the major working fluid of the system is pure water. For LHPs and CPLs, water was shown to be one of the most suitable fluids for high operation temperatures [i.e., temperatures above $70^{\circ} \mathrm{C}$ (Maydanik et al. 2014)] by comparing the measured heat transfer coefficient for different working fluids. In addition to pure water, methanol or ethanol is considered as a working fluid so as to compare and assess the effective heat transfer coefficient of a porous wick operating with a different type of working fluid. The physicalthermal properties of methanol and ethanol can be found in Faghri (1995). Due to the high wettability of these working fluids on a solid substrate, glass in this study, the equilibrium contact angle $\theta$ is assumed to be zero. 
Here, the equations of state for pure water were used according to the IAPWS Formulation 1995 (Wagner and Pruß 2002). For example, the temperature-dependent saturation vapor pressure is computed by

$$
\ln \left(\frac{p_{\text {sat }}(T)}{p_{\text {critical }}}\right)=\frac{T_{\text {critical }}+273.15}{T+273.15}\left(a_{1} v+a_{2} v^{1.5}+a_{3} v^{3}+a_{4} v^{3.5}+a_{5} v^{4}+a_{6} v^{7.5}\right)
$$

where $T$ and $T_{\text {critical }}$ denote the vapor and critical temperatures of water, respectively. $v=1-\frac{T+273.15}{T_{\text {critical }}+273.15}$ and $a_{1}, a_{2}, a_{3}, a_{4}, a_{5}, a_{6}$ denote empirically determined coefficients.

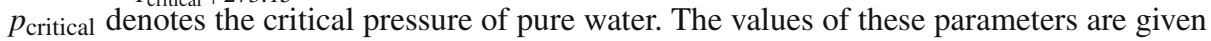
by $T_{\text {critical }}=373.95^{\circ} \mathrm{C}, p_{\text {critical }}=22.06 \mathrm{MPa}, a_{1}=-7.85, a_{2}=1.84, a_{3}=-11.78$, $a_{4}=22.68, a_{5}=-15.96$ and $a_{6}=1.80$. The equations of state for other thermal properties such as the evaporation enthalpy, viscosity, thermal conductivity, and density of liquid and vapor water can be found in Wagner and Pruß (2002). For other application such as porous burners, the equations of state for the liquid fuels involved can be used in a similar calculation.

\subsection{Numerical Algorithm}

Figure 5 illustrates the flowchart of the numerical algorithm implemented to determine the steady-state phase and temperature distributions inside a porous wick. First, the data structures of the pores and throats in the pore network are initialized. In the initialization routine, the throats in the first row underneath the fin are assumed to be empty, whereas all remaining throats are filled with liquid. The temperature field is computed by solving a system of Eq. 6 for given boundary conditions (see Eqs. 10, 12-15). After that, the thermal properties of vapor and water are updated by the equation of state for water (Wagner and Pruß 2002). The evaporation rates are determined by Eqs. 17 and 19, whereas the liquid and vapor pressure fields are obtained by solving a system of Eqs. 1-3. From the pressure field, the pressure difference $\Delta p_{i j}$ between the vapor and the liquid pores of each interface throat is computed. These values are compared with the respective values of the throat capillary pressures. The pressure difference must be equal to the capillary pressure for menisci located inside the throats. However, for a meniscus located at the open end of a throat, the pressure difference may be smaller than the capillary pressure. Due to the discretization error, we assume that the location of the stationary liquid-vapor interface has been found when $\Delta p_{i j} \leq(1+\xi) p_{c, i j}$ where $\xi$ is a convergence criterion parameter; see Table 1 . In the case where $\Delta p_{i j}>(1+\xi) p_{c, i j}$, the throat $i j$ becomes a vapor throat. After updating the phase distribution, the new liquid-vapor interface throats are determined. Then the algorithm goes back to solving the energy equation and the overall procedure is iterated. The solution has converged when $\Delta p_{i j} \leq(1+\xi) p_{c, i j}$ for all interface throats.

Because the vapor invasion within the network is mainly controlled by capillary action, the variation of the parameter $\xi$ directly impacts the phase distribution. A lower value of the $\xi$ may lead to a solution with a higher accuracy; however, it requires a longer computation time. In this sense, the parameter $\xi$ introduces a trade-off between the solution accuracy and the computational cost. As presented in "Appendix 1" the vapor area fraction varies only slightly (lower than $1 \%$ ) when $\xi$ increases from 0 to 0.01 , whereas the computational time is reduced by approximately $10 \%$. Hence, the value of 0.01 was chosen for the simulations presented in this study. 
Fig. 5 Flowchart of the numerical algorithm to determine the steady-state liquid-vapor interface inside the porous wick

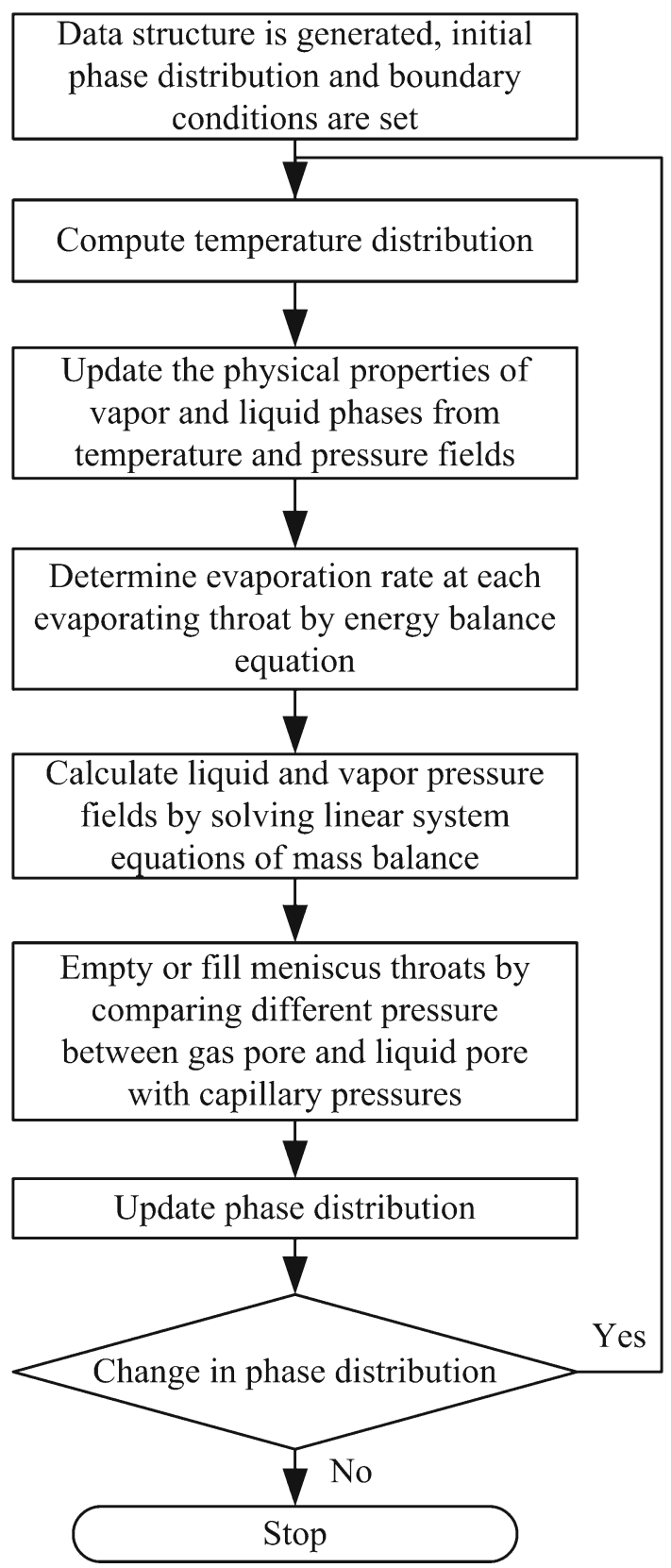

\section{Simulation Results and Discussion}

The pore network model described in Sect. 3 is used to simulate the heat and mass transfer that occur in the computational domain shown in Fig. 1 during the wicking process. The numerical solution for the temperature and pressure distributions inside this domain as well 
Table 1 Parameters used for pore network simulations

\begin{tabular}{lll}
\hline Parameter & Value & Unit \\
\hline Number of columns in the pore network & 50 & - \\
Number of rows in the pore network & 44 & - \\
Horizontal length of the wick $L_{x}$ & 49 & $\mathrm{~mm}$ \\
Vertical length of the wick $L_{y}$ & 43 & $\mathrm{~mm}$ \\
Fin length & 25 & $\mathrm{~mm}$ \\
Throat length $L$ & 1000 & $\mu \mathrm{m}$ \\
Throat radius $r$ & 70 & $\mu \mathrm{m}$ \\
Solid material & $\mathrm{Glass}$ & - \\
Thermal conductivity of solid phase $\lambda_{\mathrm{s}}$ & 10 & $\mathrm{~W} / \mathrm{mK}$ \\
Vapor temperature at vapor outlet $T_{\text {out }}$ & 100 & ${ }^{\circ} \mathrm{C}$ \\
Vapor pressure at vapor outlet $p_{\text {out }}$ & $p_{\text {sat }}\left(T_{\text {out }}\right)$ & $\mathrm{Pa}$ \\
Liquid inlet temperature $T_{\text {in }}$ & 95 & ${ }^{\circ} \mathrm{C}$ \\
Liquid inlet pressure $p_{\text {in }}$ & $p_{\text {out }}-100$ & $\mathrm{~Pa}$ \\
Convergence parameter $\xi$ & 0.01 & - \\
\hline
\end{tabular}

as for the vapor pocket boundary is given for different values of the heat load. Moreover, the influence of the heat load, the porous structure, as well as the liquid inlet pressure and temperature on the heat transfer efficiency is investigated and discussed. The geometrical, physical, and numerical parameters used in the following pore network simulations are shown in Table 1. The throat length and radius used for the simulations were taken from a physical micromodel with etched channels fabricated to be used for our future experiments. Although these throat diameters are not representative of a real porous wick structure, the parametric studies performed using the present model are still capable of capturing behaviors of the system under parameter variations.

\subsection{Model Verification}

Due to the irregular pore space structure of a real porous wick and the lack of information about the pore size distribution and throat length needed to generate a pore network, the operating characteristics of a pore network obtained from simulations cannot be fully experimentally validated. This pore network model will become predictive after further development and parametrization in well-defined experiments. Such a parametrization is not yet possible from the available data in the literature. In this work, the validity of the model is checked by comparing the heat transfer coefficient versus the heat load with experimental observations extracted from Liao and Zhao (2000). Their experiment was conducted with a porous wick structured by staggered silver-copper cylinders with a diameter of $2 \mathrm{~mm}$ and water was used as a working fluid. In this section, the pore network simulations are performed for a throat length and radius of $L=5 \mathrm{~mm}$ and $r=1 \mathrm{~mm}$, respectively. The effective heat transfer coefficient is calculated by

$$
\alpha_{\text {eff }}=\frac{\dot{q}}{T_{\text {fin }}-T_{\text {out }}}
$$

Here $T_{\text {fin }}-T_{\text {out }}$ denotes the fin superheat value, which is the difference of the temperature at the center of the fin's bottom edge, $T_{\text {fin }}$, and of the vapor temperature at the vapor outlet, $T_{\text {out }}$. Since the heat transfer efficiency is proportional to $\alpha_{\text {eff }}$, Eq. (21) implies that for a given heat load $\dot{q}$, 
Fig. 6 Effective heat transfer coefficient versus the heat load during the dry-out operating regime with $T_{\text {in }}=50^{\circ} \mathrm{C}$, $p_{\text {in }}=p_{\text {out }}-100 \mathrm{~Pa}$

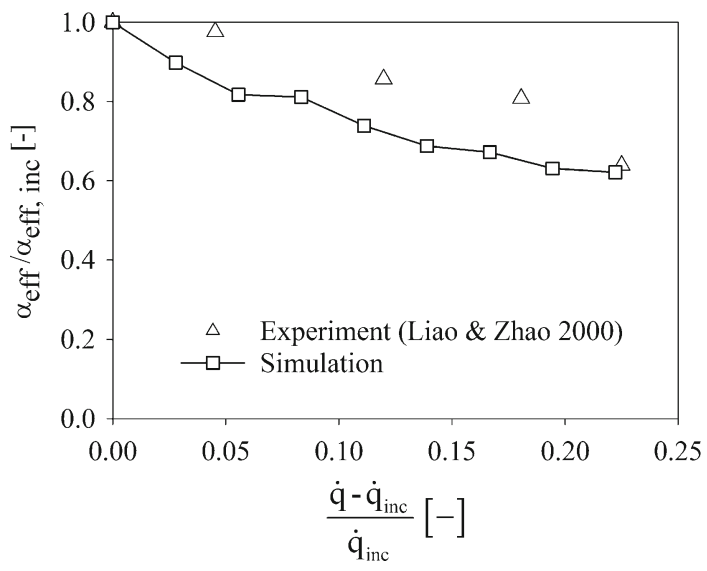

a larger fin superheat value leads to a lower heat transfer efficiency of the capillary wick. The normalization is performed by comparing the temporary effective heat transfer coefficient $\alpha_{\text {eff }}$ and the heat load $\dot{q}$ with their values at the incipient condition $\alpha_{\text {eff, inc }}$ and $\dot{q}_{\text {inc }}$ (the lowest heat load where the vapor pocket starts to be formed under the fin). According to the experimental data, the incipient point is reached when the heat transfer coefficient starts to significantly reduce. The experimental heat load $\dot{q}_{\text {inc }}$ and the effective heat transfer coefficient $\alpha_{\text {eff, inc }}$ at the incipient point are $189 \mathrm{~kW} / \mathrm{m}^{2}$ and $10,800 \mathrm{~W} / \mathrm{m}^{2} \mathrm{~K}$, respectively, where the operating conditions are $T_{\text {in }}=50^{\circ} \mathrm{C}, p_{\text {in }}=p_{\text {out }}-100 \mathrm{~Pa}$. Under these conditions, the respective values obtained from the simulation are $\dot{q}_{\text {inc }}=175 \mathrm{~kW} / \mathrm{m}^{2}$ and $\alpha_{\text {eff, inc }}=33127 \mathrm{~W} / \mathrm{m}^{2} \mathrm{~K}$. The experimental effective heat transfer coefficient is significantly lower than the numerical value because the solid phase in the experimental apparatus is not interconnected as in the pore network model. Therefore, the effective thermal conductivity and the conductive heat flux obtained for a real porous wick are smaller compared to the simulation values. The comparison between simulation and experimental observations is presented in Fig. 6. It can be seen that the simulated effective heat transfer coefficients obtained from the pore network simulations for various values of the heat load follow a trend similar to the measured data. This qualitative agreement indicates the predictive power of the model developed in the present study in the dry-out operating regime of an unsaturated capillary porous wick.

\subsection{Temperature and Pressure Distributions}

In this section, the simulations are performed with pure water as the working fluid for two cases: (a) with constant thermal properties and (b) with thermal properties depending on both temperature and pressure. In case (a), the pore network model becomes similar to the model of Figus et al. (1999). However, in this work, the number of thermal nodes is two times smaller and the evaporation at the wet wick surface is taken into account. The temperature distribution as well as the evaporation front interface for a heat load of $\dot{q}=140 \mathrm{~kW} / \mathrm{m}^{2}$ is presented in Fig. 7a. The evolution of the shape of the vapor pocket over time is qualitatively similar to previously published results (Figus et al. 1999). However, a significant increase in temperature from the evaporation front to the top center of the porous wick in Fig. 7a leads to a remarkable decrease of the vapor density, which can restrict the vapor transport. For a more realistic simulation, the dependency of the thermal properties on both temperature and pressure are taken into account [case (b)], and the simulation results are shown in Fig. 7b. 


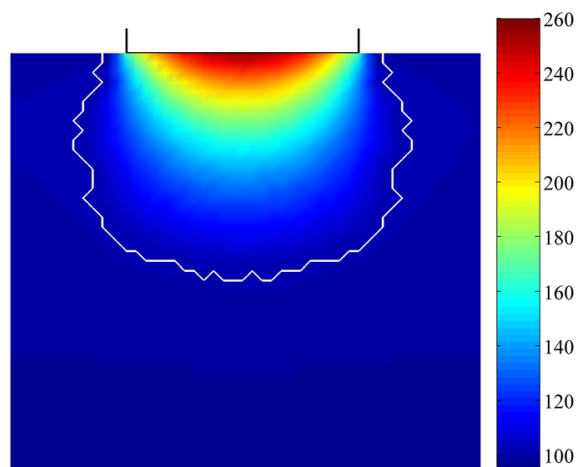

(a)

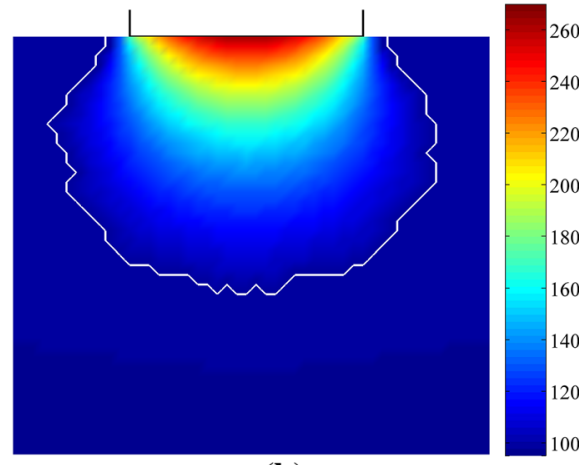

(b)

Fig. 7 Spatial distribution of temperature at steady state for $\dot{q}=140 \mathrm{~kW} / \mathrm{m}^{2}$ : a with constant thermal properties, $\mathbf{b}$ with thermal properties that depend on both temperature and pressure. The liquid-vapor interface is indicated by a white line, and the color indicates the temperature in ${ }^{\circ} \mathrm{C}$

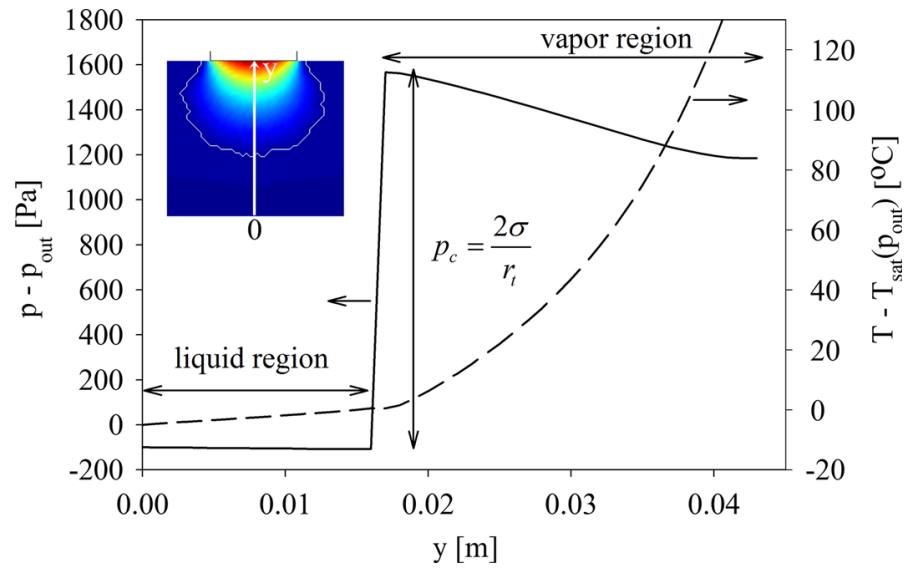

Fig. 8 Cross section of the pressure and temperature profiles in the pore network along the vertical arrow shown in the inset; $\dot{q}=140 \mathrm{~kW} / \mathrm{m}^{2}$

The area fraction of the vapor region which is defined in Eq. 22 increases from 0.32 in case (a) to 0.39 in case (b).

$$
f_{\mathrm{v}}=\frac{A_{\text {vapor pocket }}}{A_{\text {porous wick }}}
$$

The reason for this increase is that the decrease in vapor density strongly intensifies both the kinematic viscosity of the vapor phase and the hydraulic resistance in the vapor region (cf. Eq. 1). Therefore, the vapor pressure at the liquid-vapor interface increases and the liquid invades further. As a result, the size of the vapor pocket is larger in case (b) than in case (a). Vertical cross sections of the pressure and temperature profiles for case (b) are shown in Fig. 8. It can be seen that the temperature of the liquid and vapor phases at the evaporation front is equal to the saturation temperature at local vapor pressure $T_{\text {sat }}\left(p_{\mathrm{v}}\right)=100.44{ }^{\circ} \mathrm{C}$. Inside the vapor pocket, the vapor is superheated and the temperature maximum is located at the fine center. There is a discontinuity in the pressure field at the liquid-vapor interface. In the cross section (Fig. 8) it is shown that the liquid pressure decreases slightly (by $8 \mathrm{~Pa}$ ) 
within the liquid region. A large jump of the pressure occurs at the liquid-vapor interface due to the capillary pressure at the meniscus throats, where the pressure reaches its highest value. Note that although this jump is the driving force of the vapor and liquid transport, it was not shown in previous 2D pore network simulations (Figus et al. 1999). In the vapor region, the pressure decreases due to the vapor flow from the liquid-vapor interface toward the dry wick surface. The pressure drop in the vapor region is more significant than in the liquid region (329 Pa). The difference between these pressure drops can be explained by the difference in kinematic viscosity $v$ between the liquid and vapor phases (in this simulation, the average kinematic viscosity of water in the liquid region is $3.01 \times 10^{-7} \mathrm{~m}^{2} / \mathrm{s}$, whereas the kinematic viscosity of vapor varies noticeably between $2.04 \times 10^{-5} \mathrm{~m}^{2} / \mathrm{s}$ at the evaporation front and $4.50 \times 10^{-5} \mathrm{~m}^{2} / \mathrm{s}$ at the center of the fin's bottom edge). The significant variation in the kinematic viscosity of the vapor indicates that incorporating the dependency of physicalthermal properties on the temperature and pressure can improve the accuracy of the model, especially for large heat load values, where a huge temperature gradient appears (see Fig. 6 for instance). Therefore, this dependency is taken into account in the following computations.

A supplementary investigation on the convective heat transfer and on the viscous dissipation is addressed in "Appendix 2". With the heat load $\dot{q}=140 \mathrm{~kW} / \mathrm{m}^{2}$, the average interstitial velocity $v_{0}$ of the vapor and liquid phases is $0.45 \mathrm{~m} / \mathrm{s}$ and $2.80 \times 10^{-4} \mathrm{~m} / \mathrm{s}$, whereas the average superficial velocity $v$ of these phases is $6.90 \times 10^{-3} \mathrm{~m} / \mathrm{s}$ and $4.30 \times 10^{-6} \mathrm{~m} / \mathrm{s}$, respectively. The convective heat flux in the vapor and liquid regions is only 0.08 and $0.18 \%$ of the conductive heat flux, respectively. Furthermore, the temperature increase due to viscous heating in vapor and liquid flows in throats with vertical dimension $L_{y}=0.044 \mathrm{~mm}$ is only $0.51 \mathrm{~K}$ and $1.47 \times 10^{-06} \mathrm{~K}$, respectively; these values are much smaller than the fin superheat value at operating condition, $T_{\text {fin }}-T_{\text {out }}=164.32 \mathrm{~K}$. This implies that both convective heat transfer and viscous dissipation are truly negligible in the present pore network configurations.

\subsection{Influence of the Heat Load on the Shape of the Vapor Pocket and on the Heat Transfer Efficiency}

The effect of the heat load on the heat and mass transfer inside the porous wick is investigated in this section. The pore network simulations are carried out with the heat load $\dot{q}$ varying from 20 to $163 \mathrm{~kW} / \mathrm{m}^{2}$, and the simulation results are presented in Figs. 9 and 10 . At $\dot{q}=20 \mathrm{~kW} / \mathrm{m}^{2}$, the vapor pocket appears underneath the fin, where the vapor only occupies $2.72 \%$ of the porous area (Fig. 9a). As the heat load increases to $60 \mathrm{~kW} / \mathrm{m}^{2}$, the vapor pocket primarily expands toward the network bottom (Fig. 9b) while the vapor reaches the outlet through a narrow neck. With further increasing heat load, several liquid throats near the neck empty and thus the vapor pocket starts to expand in the lateral direction. This leads to a decrease of the pressure inside the vapor pocket and several empty throats are refilled at the same time (Fig. 9c), such that the vapor pocket shrinks. As the heat load increases further, the vapor pocket expands again (Fig. 9d). The lengths of the dry edges DE and FG at the wick surface (cf. Fig. 1) remain unchanged because the vapor outflow is not strong enough to invade the liquid throats at the surface. With an even higher heat load, the liquid throats at the surface are finally invaded and the hydraulic resistance of the vapor phase decreases again. Therefore, the vapor pressure inside the pocket drops and several empty throats are refilled by liquid, which leads to a decrease of the vapor pocket size (Fig. 9e). This inflation and deflation behavior of the vapor pocket repeats with further increasing heat load (Fig. 9f).

The influence of the heat load on the effective heat transfer coefficient is shown in Fig. 9. As the heat load increases, the distance between the heat source (fin) and the liquid-vapor interface inside the porous wick increases first and then fluctuates. Consequently, the thermal 


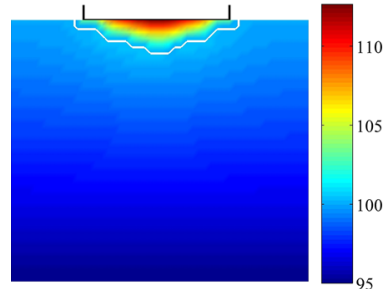

(a)

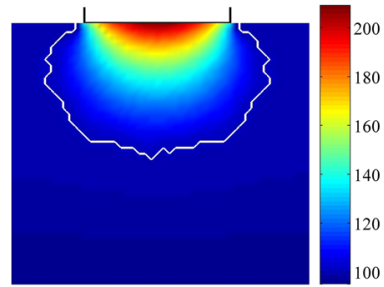

(d)

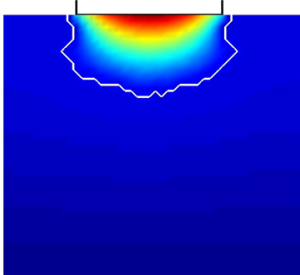

(b)

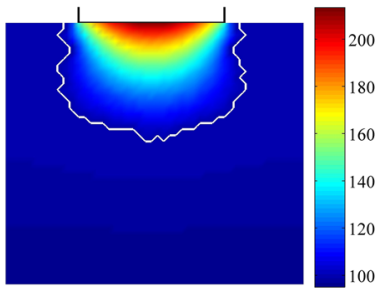

(e)

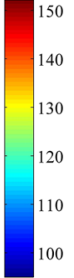

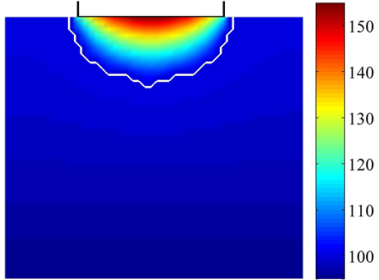

(c)

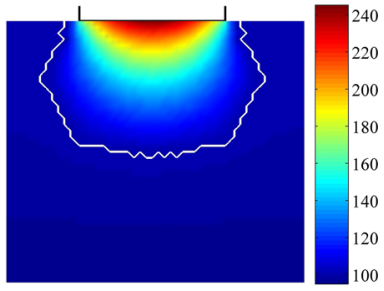

(f)

Fig. 9 Temperature distribution for different values of the heat load: $\mathbf{a} \dot{q}=20 \mathrm{~kW} / \mathrm{m}^{2}, \mathbf{b} \dot{q}=60 \mathrm{~kW} / \mathrm{m}^{2}$, c $\dot{q}=70 \mathrm{~kW} / \mathrm{m}^{2}, \mathbf{d} \dot{q}=100 \mathrm{~kW} / \mathrm{m}^{2}$, e $\dot{q}=110 \mathrm{~kW} / \mathrm{m}^{2}, \mathbf{f} \dot{q}=130 \mathrm{~kW} / \mathrm{m}^{2}$ (color indicates the temperature in ${ }^{\circ} \mathrm{C}$ )

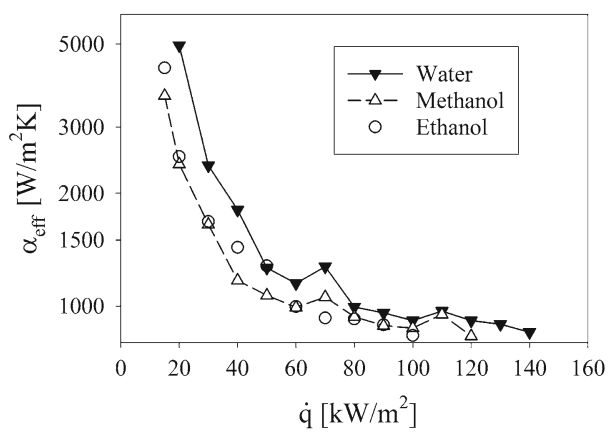

(a)

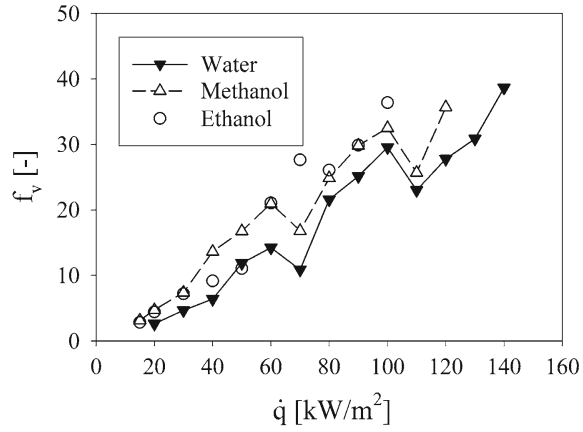

(b)

Fig. 10 Influence of the heat load $\dot{q}$ on the effective heat transfer coefficient $\alpha_{\text {eff }}$ and on the area fraction of the vapor region $f_{\mathrm{V}}$

resistance between the fin and the evaporation front increases first-due to the lower thermal conductivity in the vapor phase - and then fluctuates. To quantify the evolution of the vapor pocket for increasing heat load more precisely, the area fraction of the vapor region $f_{\mathrm{v}}$ is calculated and shown in Fig. 10. For a given value of the heat load $\dot{q}$, the area fraction of the vapor region $f_{\mathrm{v}}$ is defined as the vapor area divided by the total area of the porous wick. One can clearly see that as the heat load increases, the area fraction of the vapor region fluctuates due to the inflation/deflation of the vapor pocket described before. The influence of the heat load on the Péclet number and on the viscous dissipation in the case where water is used as a working fluid is presented in detail in "Appendix 2".

The simulation results obtained from three different types of working fluids are shown in Fig. 10. Considering on the vapor area fraction, the inflation/deflation phenomenon is also observed with both methanol and ethanol. Due to a lower evaporation enthalpy of methanol 
Fig. 11 Influence of the throat radius $r$ on the area fraction of the vapor region $f_{\mathrm{V}}$ at $\Delta p=400 \mathrm{~Pa}$

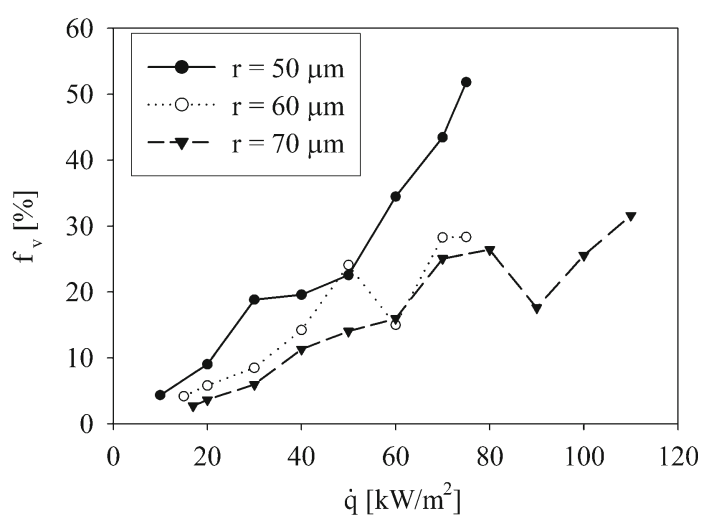

and of ethanol compared to water (the evaporation enthalpies of methanol, ethanol, and water at $100{ }^{\circ} \mathrm{C}$ are 1030,927 , and $2251 \mathrm{~kJ} / \mathrm{kg}$, respectively), the evaporation rate obtained with methanol and ethanol is smaller compared to water. Although the kinematic viscosity of methanol or of ethanol is higher than that of water, the higher evaporation rate and lower surface tension lead to a further invasion of vapor phase. As a result, the gap between the fin and the evaporation front increases and the effective heat transfer coefficient decreases. These results favor water as the most effective working fluid at high temperature for LHPs and CPLs (Maydanik 2005).

The inflation/deflation phenomenon observed in the simulations may explain the fluctuation of the heat transfer coefficient found in experimental studies (Liao and Zhao 1999, 2000; Zhao and Liao 2000). However, this phenomenon still needs to be confirmed by sophisticated pore-scale experiments. Optical microfluidic models or X-ray microtomography shall be used to visualize and quantify this phenomenon in future work.

\subsection{Influence of the Porous Structure on the Heat Transfer Efficiency}

\subsubsection{Discrete and Continuous Unimodal Throat Radius Distributions}

To examine the heat transfer efficiency of different porous structures, the situation of a constant throat radius was explored first. Note that water is used as the working fluid throughout the remaining sections. The simulations are performed for throat radii of $r=50,60,70 \mu \mathrm{m}$ and for pressure differences of $\Delta p=p_{\text {out }}-p_{\text {in }}=400 \mathrm{~Pa}$. The results are presented in Figs. 11 and 12. As shown in Fig. 11, for a constant heat load and pressure difference, the vapor area fraction (i.e., the vapor pocket size at steady state) increases when the throat radius decreases. The primary reason for this vapor pocket expansion is the influence of the throat radius on both the capillarity and the hydraulic resistance. For a decreasing throat radius, the pressure drop in both liquid and vapor transports increases. As a result, the pressure difference between vapor and liquid pressures, $p_{\mathrm{v}}-p_{1}$, at the evaporation front increases with a decreasing throat radius. Because the hydraulic resistance due to the viscous force more strongly depends on the throat radius than on the capillary pressure, the pressure difference at the liquid-vapor interface increases faster than the capillary threshold for a decreasing throat radius: $p_{\mathrm{v}}-p_{\mathrm{l}}>p_{\mathrm{c}}$. It means that the vapor invades further into the network with smaller throat radii resulting in a bigger vapor pocket. This vapor pocket expansion helps to decrease the difference of vapor and liquid pressures at the liquid-vapor interface to balance 
Fig. 12 Influence of the throat radius $r$ on the fin superheat value $T_{\text {fin }}-T_{\text {out }}$ at $\Delta p=400 \mathrm{~Pa}$

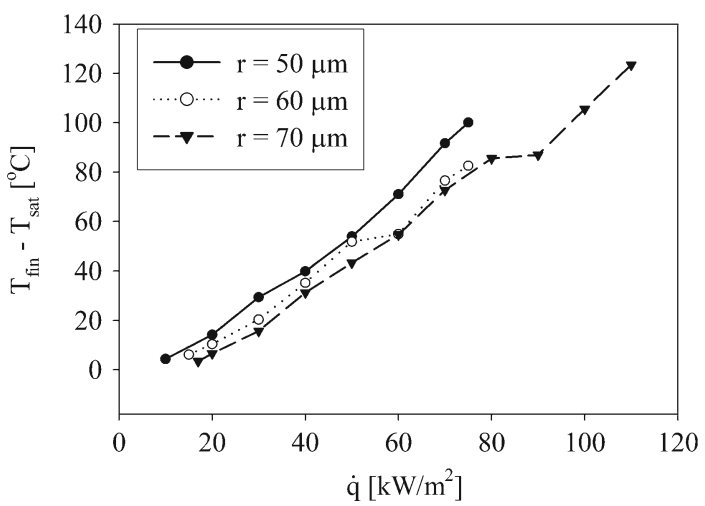

the capillary force by reducing the length of the flow path of the liquid phase (increasing the liquid pressure) and increasing the dry part of the vapor outlet (edges DE and FG in Fig. 1) (decreasing the vapor pressure). Since the gap between the fin and the evaporation front increases during the vapor pocket expansion, the fin superheat value increases with decreasing throat radius, as shown in Fig. 12. However, for certain heat load values $\left(\dot{q}=50 \mathrm{~kW} / \mathrm{m}^{2}\right.$ and $\dot{q}=60 \mathrm{~kW} / \mathrm{m}^{2}$ in Fig. 12), this general observation is not true. These exceptions can be explained by the inflation/deflation phenomenon described in Sect. 4.3. The distance between the hot surface and the evaporation front fluctuates with increasing heat load, which leads to a fluctuation of the fin superheat value.

In reality, the porous structure is neither homogeneous nor isotropic. In this case, a network with a uniform throat diameter is not representative of a real porous wick. The results obtained for a uniform throat radius are nonetheless useful because they show the benefit of using throats with variable size. In order to understand the pore-scale heat and mass transfer for a more realistic structure, the throat radii are sampled from a normal distribution with mean $r_{0}$, and standard deviation $\sigma_{0}$ in the next example.

$$
\frac{\mathrm{d} N}{\mathrm{~d} r}=\frac{1}{\sigma_{0} \sqrt{2 \pi}} e^{-\frac{\left(r-r_{0}\right)^{2}}{2 \sigma_{0}^{2}}}
$$

The mean throat radius is set to $r_{0}=70 \mu \mathrm{m}$, whereas the standard deviation $\sigma_{0}$ varies from $2 \% r_{0}$ to $20 \% r_{0}$. The simulation results for $\dot{q}=80 \mathrm{~kW} / \mathrm{m}^{2}$ are shown in Fig. 13 . The morphology of the evaporation front changes significantly with the standard deviation of the throat radius distribution. In comparison with the previous cases with constant throat size, the liquid is more easily pumped from the bottom to the evaporation front in small throats (due to the high capillary pressures). Furthermore, large throats are more easily invaded by the vapor phase due to lower capillary thresholds. Note that the average permeability of the network depends only on the mean throat radius (not on the standard deviation). In this case, the evaporation area is given by the large throats, whereas the liquid is pumped through the small throats from the inlet to the evaporation front. This effect is more pronounced for a broader throat radius distribution (higher standard deviation), such that several capillaryviscous vapor fingers appear at the evaporation front. This (fractal) behavior of the interface, as in a typical invasion process, is determined by the interplay between viscous and capillary forces (Metzger et al. 2014).

Because small throats near the fin can hardly be invaded by the vapor phase, the area fraction of the vapor region decreases (from $24.81 \%$ with $\sigma_{0}=0$ to $14.63 \%$ with $\sigma_{0}=$ 


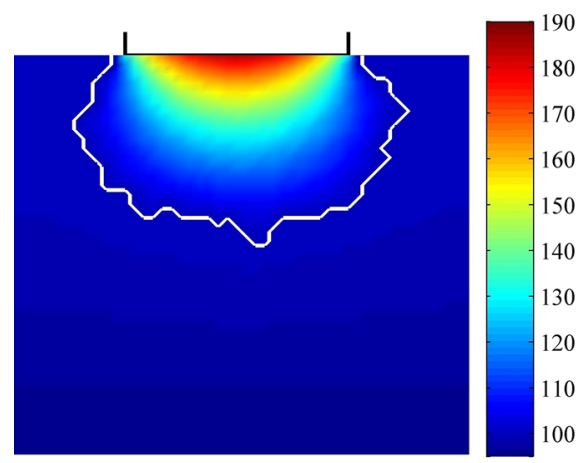

(a)

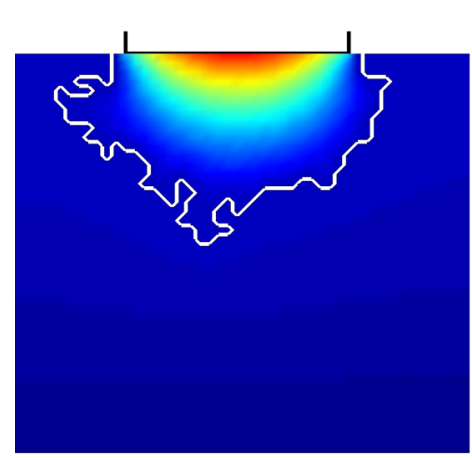

(c)

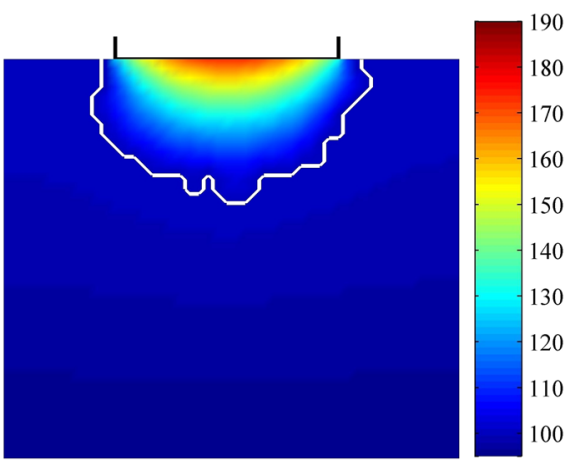

(b)

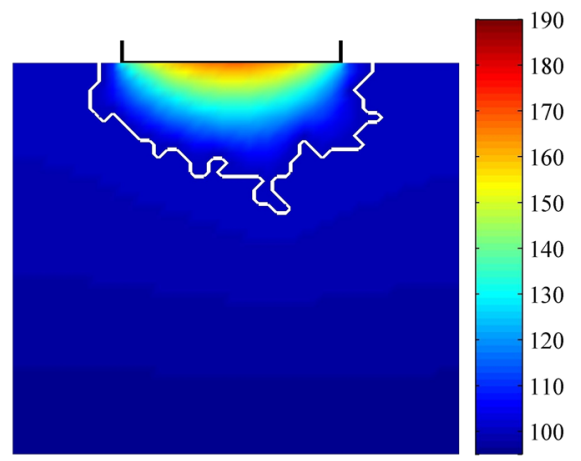

(d)

Fig. 13 Morphology of the liquid-vapor interface and the temperature distribution in the porous wick for different values of the standard deviation of the unimodal throat radius distribution $\mathbf{a} \sigma_{0}=2 \% r_{0}$, b $\sigma_{0}=$ $5 \% r_{0}, \mathbf{c} \sigma_{0}=10 \% r_{0}$, and $\mathbf{d} \sigma_{0}=20 \% r_{0}$ at $\dot{q}=80 \mathrm{~kW} / \mathrm{m}^{2}$ and $\Delta p=400 \mathrm{~Pa}$ (color indicates the temperature in ${ }^{\circ} \mathrm{C}$ )

$20 \% r_{0}$ ) for increasing standard deviation. Therefore, the fin superheat value decreases from $84.14{ }^{\circ} \mathrm{C}$ with $\sigma_{0}=2 \% r_{0}$ to $67.77^{\circ} \mathrm{C}$ with $\sigma_{0}=20 \% r_{0}$ (note that the fin superheat value for the uniform throat size $r=70 \mu \mathrm{m}$ is $85.53^{\circ} \mathrm{C}$ ). This implies that the heat transfer efficiency is enhanced for a non-constant throat size.

\subsubsection{Continuous Bimodal Throat Radius Distribution}

From the simulation results for pore networks with unimodal throat radius distributions, it was conceived that a pore network with a bimodal throat radius distribution might further enhance the heat and mass transfer in the porous wick. In this paper, a pore network with a bimodal throat radius distribution was generated, containing vertical macrochannels (cf. Fig. 14). While the network of small throats provides a high liquid pump capacity, the network of large throats acts as an evaporative heat exchanger. This idea was shown to work in a biporous wick (Cao et al. 2002; Byon and Kim 2012); a theoretical explanation is not given in the literature, however.

The pore network simulations are performed for a network with bimodal throat radius distributions. In the network with constant throat size $r=70 \mu \mathrm{m}$, macrochannels with a radius of $r_{\mathrm{ch}}=1.5 \mathrm{r}$ are inserted in every third column. The simulation results for a pressure 


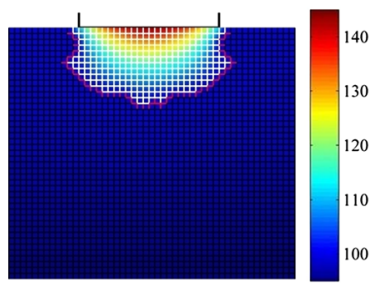

(a)

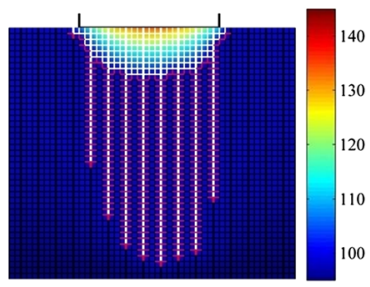

(b)

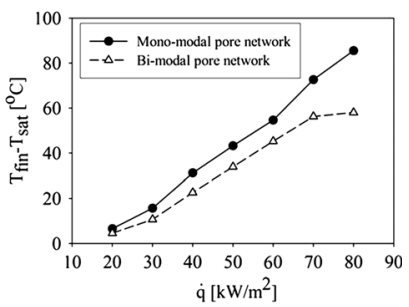

(c)

Fig. 14 Temperature distributions for a a unimodal pore network and $\mathbf{b}$ a bimodal pore network at $\dot{q}=$ $80 \mathrm{~kW} / \mathrm{m}^{2}$ and $\Delta p=400 \mathrm{~Pa}$ (color indicates the temperature in ${ }^{\circ} \mathrm{C}$, black lines liquid throats, white lines vapor throats, and red lines partially filled throats). c Influence of the heat load $\dot{q}$ on the fin superheat value $T_{\text {fin }}-T_{\text {out }}$ in unimodal and bimodal pore networks at $\Delta p=400 \mathrm{~Pa}$

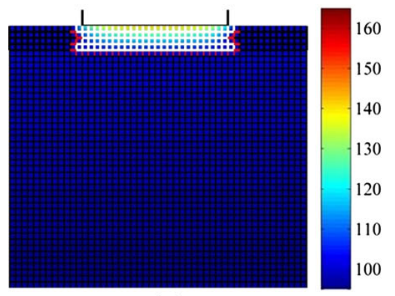

(a)

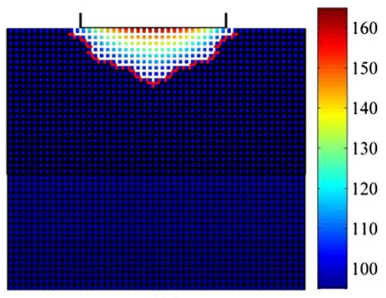

(b)

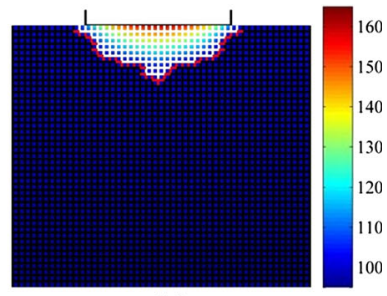

(c)

Fig. 15 Temperature distributions for bilayer porous wicks with relative coarse layer thickness a $e=9.3 \%$, b $e=55.8 \%$, and $\mathbf{c} e=100 \%$ at $\dot{q}=100 \mathrm{~kW} / \mathrm{m}^{2}$ and $\Delta p=400 \mathrm{~Pa}$ (black lines liquid throats, white lines vapor throats, and red lines partially filled throats; color indicates the temperature in ${ }^{\circ} \mathrm{C}$ )

difference of $\Delta p=400 \mathrm{~Pa}$ are presented in Fig. 14a, b. It can be seen that the macrochannels are preferentially invaded by the vapor phase due to lower entry capillary thresholds $p_{\mathrm{c}}$ (cf. Eq. 18). Several vapor channels develop in the pore network (Fig. 14b), and the vapor pocket is smaller compared to the pore network with a unimodal pore radius distribution (Fig. 14a). The liquid and vapor flows are separated: The liquid is transported through the small throats by capillary action, whereas the vapor escapes through the macrochannels, as expected. Because the vapor pocket is smaller, the distance between the heat source and the liquid-vapor interface is reduced compared to the network with the unimodal pore radius distribution. Therefore, the fin superheat value is also smaller (Fig. 14c).

\subsubsection{Bilayer Pore Network Structures}

Another idea to enhance the heat and mass transfer inside the porous wick is to insert an additional porous layer with large throat radii on top of the current porous wick. Again, the finer porous layer would operate as a capillary pump at the bottom while the coarser porous layer would act as an evaporative heat exchanger at the top. This idea was introduced in the work of Mottet et al. (2014); in the present paper, the effect of the coarse layer thickness is evaluated and quantified via pore network simulations.

Starting with the original unimodal pore network (Sect. 4.4a), the radii of several throats near the top of the network were increased by a factor of 1.5 . The thickness of the resulting coarse layer is denoted as $L_{\text {coarse }}$, and $e=L_{\text {coarse }} / L_{y}$ denotes the relative coarse layer thickness. Simulations are performed for several values of $e$ ranging from 9.3 to $100 \%$, and the corresponding results are presented in Figs. 15 and 16. Note that for $e=0 \%$, the pore 
Fig. 16 Influence of the heat load $\dot{q}$ on the fin superheat value $T_{\text {fin }}-T_{\text {out }}$ for bilayer pore networks at $\Delta p=400 \mathrm{~Pa}$

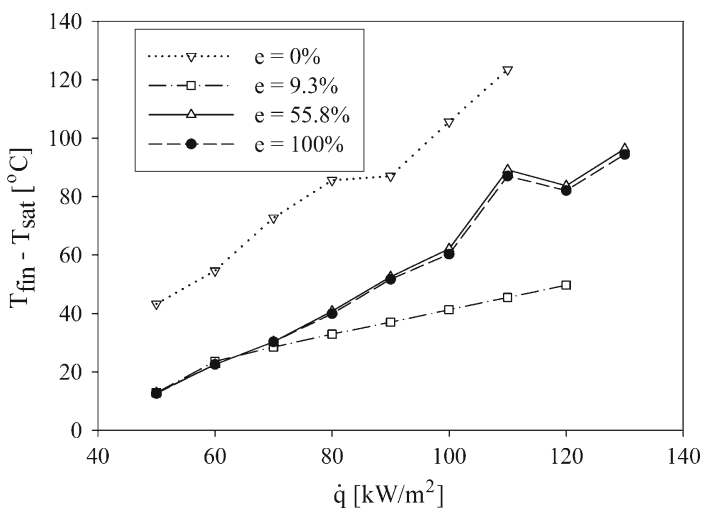

Table 2 A summary of the pore network simulation results

\begin{tabular}{llll}
\hline Pore network & Parameter & $\begin{array}{l}\text { Fin superheat } \\
\text { value } T_{\text {fin }}-T_{\text {out }}\end{array}$ & $\begin{array}{l}\text { Area fraction of } \\
\text { the vapor region } f_{\mathrm{V}}\end{array}$ \\
\hline Unimodal & $\begin{array}{l}\text { Mean throat radius, } r_{0} \uparrow \\
\text { (Figs. 11, 12) }\end{array}$ & $\downarrow$ & $\downarrow$ \\
& Standard deviation, $\sigma_{0} \uparrow$ (Fig. 13) & $\downarrow$ & $\downarrow$ \\
Bimodal & Relatively to unimodal pore network & $\downarrow$ & $\downarrow$ \\
Bilayer & (Fig. 14) & First $\downarrow$ and $\uparrow$ then & First $\downarrow$ and $\uparrow$ then \\
& Relative thickness of coarse layer $e \uparrow$ & &
\end{tabular}

“个” indicates an increase, “_” indicates no or only a slight change, and “ $\downarrow "$ indicates a reduction

network is unimodal with only small throats, whereas for $e=100 \%$, the pore network becomes unimodal with only large throats. It can be seen that for small values of $e$ (Fig. 15a), the vapor pocket touches the surface of the fine porous layer below. The high capillary effects of the fine layer at the liquid-vapor interface increase the pump capacity of the porous wick. Therefore, the liquid is supplied from the bottom up to the macrochannels. The depth of the vapor pocket is smaller compared to the unimodal pore network (Fig. 13a), which leads to a higher heat and mass transfer efficiency as well as to a significantly smaller fin superheat value (Fig. 16). However, if the thickness of the coarse layer becomes larger ( $e=55.8 \%$ ), the vapor pocket does not touch the fine porous layer anymore (Fig. 15b). It means that the fine layer does not impact the capillarity. In this case, the liquid flow is restrained due to a lower permeability of fine layer and thus the fin superheat value and vapor pocket size become larger compared to the case of $e=100 \%$. However, as presented in Fig. 8, the pressure drop is liquid transport is quite small compare to the pressure drop in vapor transport, the changing of liquid pressure due to the fine compartment slightly influences on the pressure profile. Thus, the vapor pocket seems identically and the heat transfer efficiency with the network of $e=55.8 \%$ and $e=100 \%$ is slightly different. With $e=100 \%$ (Fig. 15c), a unimodal pore network with macrochannels provides a higher heat and mass transfer efficiency than a unimodal pore network with microchannels (with $e=0 \%$ ). This result also matches with the results presented in Sect. 4.4a.

The major conclusions from the pore network simulations described in this section are summarized in Table 2 . The results from this section might be valid in an individual unsat- 
urated capillary porous wick such as in porous burners, whereas a coupling between the evaporator and the remaining components of LHPs and CPLs is required to investigate the operation characteristics of a complete closed loop. Very recently, Boubaker and Platel (2015) showed numerically that the size of the vapor pocket is nearly independent of the applied heat load in a complete loop of heat pipes. This observation still needs to be experimentally confirmed. It may stem from the coupled effects of thermal and hydrodynamic mechanisms involved in the interactions between the evaporator and the rest of the loop. Due to the high computational cost, this coupling is not yet included in our work. In future, both experimental and theoretical studies shall be carried out to evaluate the heat and mass transfer efficiencies of LHPs and CPLs in a fully coupled system.

\section{Conclusions}

In this paper, a pore network model was developed to simulate the heat and mass transfer in an unsaturated capillary porous wick and to predict the location of the liquid-vapor interface over time as well as the effective heat transfer coefficient in the dry-out regime. The dependence of the thermal properties of the working fluid on both temperature and pressure is taken into account in our model. The present model is verified against measurement data available in the literature. Deflation/inflation of the vapor pocket and a fluctuation of the heat transfer coefficient with increasing heat load, which cannot be obtained from continuum simulations, were observed in pore networks with unimodal throat radius distributions. By evaluating the values of Péclet number and the temperature gradient due to viscous heating, both the heat transfer by convection and the viscous dissipation are negligible in the present network configurations. A brief comparative assessment of the effective heat transfer coefficient of the porous wick operating with different types of working fluids was also implemented. The results favor water as the most effective working fluid because it has a better heat transfer performance than methanol or ethanol at high operating temperature. An enhancement of the heat and mass transfer efficiencies of a porous wick is achieved by using a bimodal throat radius distribution (with vertical macro channels) or by a layered structure (a thin coarse layer above a fine layer). However, if the relative thickness of the coarse layer is too large, then the heat transfer efficiency is reduced again.

In the future, microfluidic experiments shall be carried out to validate these simulation results. These measurements shall contribute to the identification of transport mechanisms at the pore level which could be incorporated later on into a pore network model. For the application in an LHP evaporator the current pore network model for the evaporator shall be coupled with models for other LHP components to simulate their operating characteristics as well as the influence of operating conditions on the heat transfer efficiency of LHPs.

Acknowledgments The first author is grateful for financial support from the Ministry of Education and Training (MOET) of the Socialist Republic of Vietnam (Project No. 911). The authors also express gratitude to anonymous reviewers for their contribution in order to improve this paper.

\section{Appendix 1: Influence of the Convergence Parameter $\xi$ (cf. Sect. 3.5) on the Vapor Pocket Pattern and on the Computation Time}

The simulations are performed for four different values of the convergence parameter $\xi$. All other parameters used in the simulations are given in Table 1. Figure 17 shows the dependency of the normalized computation time $\tau$ and of the vapor area fraction $f_{\mathrm{v}}$ on the values of $\xi$. 
Fig. 17 Influence of convergence parameter $\xi$ on the vapor area fraction and on the normalized computation time $\tau$. The inset figure shows the vapor pocket patterns obtained from simulations with different values of $\xi$

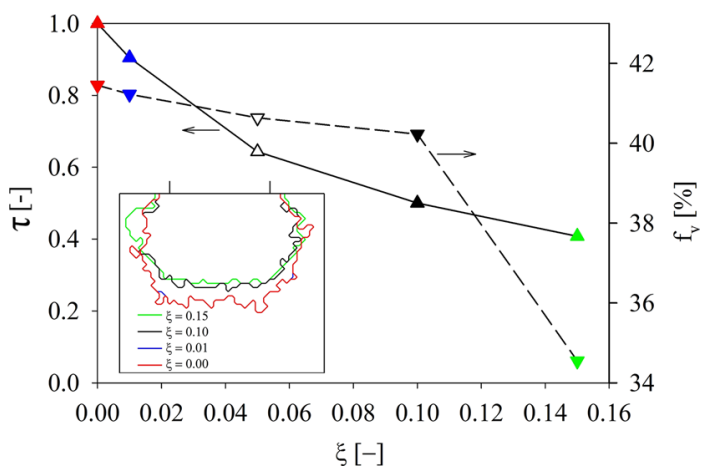

The normalized time is defined as the ratio of the time required for the simulation with a given value of $\xi$ to the simulation time with $\xi=0.00$, which varies from 4 to $7 \mathrm{~h}$ depending on throat size distribution. Due to less iterations for a larger value of $\xi$, the computation time can be reduced by about $10 \%$ by increasing $\xi$ from 0.00 to 0.01 . Since the results obtained for these two values of $\xi$ do not deviate too much, $\xi=0.01$ is chosen for all simulations presented in this study. As can also be seen, the vapor pocket patterns obtained for the values of $\xi=0.00$ and 0.01 slightly differ.

\section{Appendix 2: Supplementary Investigation on Convective Heat Transfer and Viscous Dissipation}

To develop the present model, several simplifications are made. The convective heat transfer and the viscous dissipation are assumed to be negligible. In this appendix, a supplementary investigation is made to examine the validity of these simplifications. The simulations are performed with water as a working fluid and with the input parameters stated in Table 1. From the pressure and phase distributions, the mass flow rate $\dot{M}_{i j}$ in each throat is calculated by Eq. 1 . The fluid interstitial velocity in each throat is computed as

$$
v_{0, i j}=\frac{\dot{M}_{i j}}{\rho_{\mathrm{f}} \pi r_{i j}^{2}}
$$

With the transfer area between two neighboring pores $A_{\mathrm{cv}, i j}=W L$, the superficial velocity $v$ is calculated by Eq. 25 .

$$
v_{i j}=\frac{v_{0, i j} \pi r_{i j}^{2}}{W L}
$$

To evaluate the contribution of heat transported by convection to the total heat flux, the Péclet number $P e$, which is a dimensionless value representing the ratio between convective heat transfer and conductive heat transfer is used.

$$
P e=\frac{\text { convective heat flux }}{\text { conductive heat flux }} \approx \frac{v \rho_{\mathrm{f}} c_{p, f}}{\lambda_{\text {eff }} / L}
$$

The superficial velocity used in this calculation is the average value for the vapor and liquid regions. The evolution of the Péclet number in vapor and liquid regions for different values of the heat load $\dot{q}$ is presented in Fig. 18. As can be seen, the Péclet number in both 
Fig. 18 Variation of the averaged Péclet number for different values of the heat load $\dot{q}$

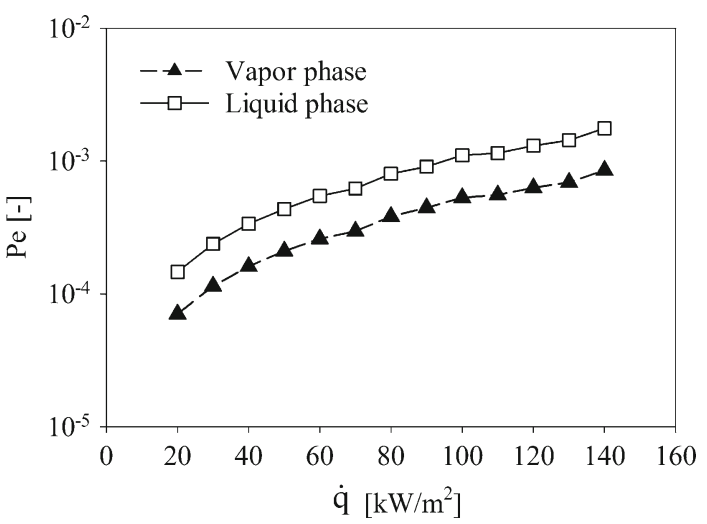

Fig. 19 Variation of the temperature gradient due to viscous heating for different values of the heat load $\dot{q}$

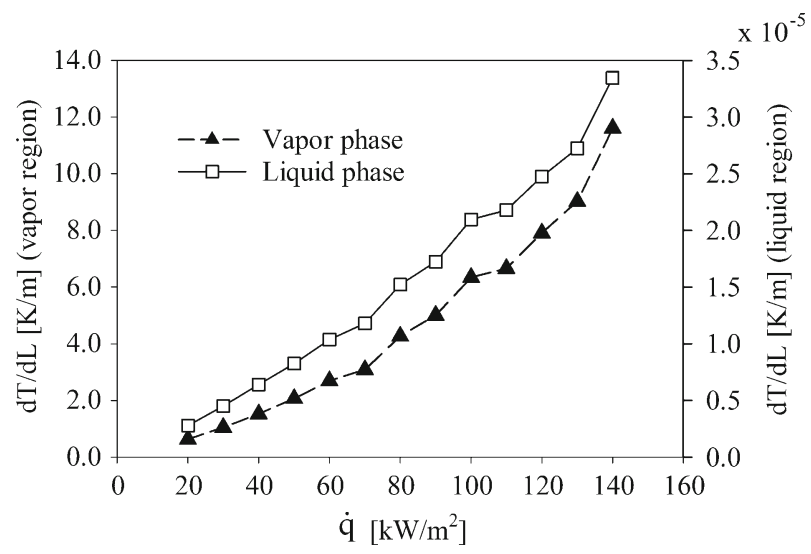

vapor and liquid regions is very small (lower than 0.002). It implies that the convective heat transfer is very small (approximately $0.2 \%$ ) compared to the conductive heat transfer, and thus the heat transported by convection is negligible in the heat flux calculations with the network configurations used in these simulations. This result also indicates that with the wick composed of non-insulated solid material, i.e., $\lambda_{\mathrm{s}}>1 \mathrm{~W} / \mathrm{mK}$, the heat transported by convection can be neglected (lower than $2 \%$ ). However, in the case of a solid skeleton made by low thermal conductivity material such as a polymer or plastic (for which the thermal conductivity varies from 0.5 to $0.2 \mathrm{~W} / \mathrm{m} \mathrm{K}$ ), the contribution of the convective heat transfer to the total heat flux is significant and this term should be considered in a more comprehensive pore network model.

In viscous flow, the mechanical energy is converted into thermal energy by viscous dissipation; thus, the temperature of the fluid increases. Morini (2005) and Han and Lee (2007) proposed an equation to compute the fluid temperature gradient along a smooth cylindrical throat due to the viscous heating as follows:

$$
\frac{\mathrm{d} T}{\mathrm{~d} L}=\frac{8 v_{o} v_{\mathrm{f}}}{c_{p, f} r^{2}}
$$

The computational results are presented in Fig. 19. The temperature gradient in the vapor flow is significantly higher than in the liquid flow because of the higher kinematic viscosity and lower density. The temperature gradient in vapor throats varies from 0.63 to $11.61 \mathrm{~K} / \mathrm{m}$ 
in a heat load range from $\dot{q}=20 \mathrm{~kW} / \mathrm{m}^{2}$ to $\dot{q}=140 \mathrm{~kW} / \mathrm{m}^{2}$. The maximum increase of the vapor temperature (for a heat load of $\dot{q}=140 \mathrm{~kW} / \mathrm{m}^{2}$ ) due to the viscous heating in a throat with length of $L=1 \mathrm{~mm}$ and $\mathrm{L}_{\mathrm{y}}=44 \mathrm{~mm}$ is 0.011 and $0.51 \mathrm{~K}$, respectively. These temperature differences are extremely small compared to the fin superheat value (164.32 K) at these operating conditions. Therefore, the influence of viscous dissipation on the fluid flow is truly negligible in the pore network configurations used in this study.

\section{References}

Boubaker, R., Platel, V., Berges, A., Bancelin, M., Hannezo, E.: Dynamic model of heat and mass transfer in an unsaturated porous wick of capillary pumped loop. Appl. Therm. Eng. 76, 1-8 (2015)

Boubaker, R., Platel, V.: Vapor pocket behavior inside the porous wick of a capillary pumped loop for terrestrial application. Appl. Therm. Eng. 84, 420-428 (2015)

Byon, C., Kim, S.J.: Effects of geometrical parameters on the boiling limit of bi-porous wicks. Int. J. Heat Mass Transf. 55(25-26), 7884-7891 (2012)

Cao, X.L., Cheng, P., Zhao, T.S.: Experimental study of evaporative heat transfer in sintered copper bidispersed wick structures. J. Thermophys. Heat Transf. 16(4), 547-552 (2002)

Cao, Y., Faghri, A.: Conjugate analysis of a flat-plate type evaporator for capillary pumped loops with threedimensional vapor flow in the groove. Int. J. Heat Mass Transf. 37(3), 401-409 (1994a)

Cao, Y., Faghri, A.: Analytical solutions of flow and heat transfer in a porous structure with partial heating and evaporation on the upper surface. Int. J. Heat Mass Transf. 37(10), 1525-1533 (1994b)

Chernysheva, M.A., Maydanik, Y.F.: 3D-model for heat and mass transfer simulation in flat evaporator of copper-water loop heat pipe. Appl. Therm. Eng. 33-34, 124-134 (2012)

Demidov, A.S., Yatsenko, E.S.: Investigation of heat and mass transfer in the evaporation zone of a heat pipe operating by the 'inverted meniscus' principle. Int. J. Heat Mass Transf. 37(14), 2155-2163 (1994)

Faghri, A.: Heat Pipe Science and Technology. Taylor \& Francis, Washington (1995)

Figus, C., Bray, Y.L., Bories, S., Prat, M.: Heat and mass transfer with phase change in a porous structure partially heated: continuum model and pore network simulations. Int. J. Heat Mass Transf. 42(14), 2557-2569 (1999)

Han, D., Lee, K.-J.: Viscous dissipation in micro-channels. J. Mech. Sci. Technol. 21(12), 2244-2249 (2007)

Jugjai, S., Phothiya, C.: Liquid fuels-fired porous combustor-heater. Fuel 86(7-8), 1062-1068 (2007)

Kaya, T., Goldak, J.: Numerical analysis of heat and mass transfer in the capillary structure of a loop heat pipe. Int. J. Heat Mass Transf. 49(17-18), 3211-3220 (2006)

Li, J., Peterson, G.P.: 3D heat transfer analysis in a loop heat pipe evaporator with a fully saturated wick. Int. J. Heat Mass Transf. 54(1-3), 564-574 (2011)

Liao, Q., Zhao, T.S.: A visual study of phase-change heat transfer in a two-dimensional porous structure with a partial heating boundary. Int. J. Heat Mass Transf. 43(7), 1089-1102 (2000)

Liao, Q., Zhao, T.S.: Evaporative heat transfer in a capillary structure heated by a grooved block. J. Thermophys. Heat Transf. 13(1), 126-133 (1999)

Louriou, C., Prat, M.: Pore network study of bubble growth by vaporization in a porous medium heated laterally. Int. J. Therm. Sci. 52, 8-21 (2012)

Maydanik, Y.F.: Loop heat pipes. Appl. Therm. Eng. 25(5-6), 635-657 (2005)

Maydanik, Y.F., Chernysheva, M.A., Pastukhov, V.G.: Review: loop heat pipes with flat evaporators. Appl. Therm. Eng. 67(1-2), 294-307 (2014)

Metzger, T., Tsotsas, E., Prat, M.: Pore-network models: a powerful tool to study drying at the pore level and understand the influence of structure on drying kinetics. In: Tsotsas, E., Mujumdar, A.S. (eds.) Modern Drying Technology, pp. 57-102. Wiley-VCH Verlag GmbH \& Co. KGaA, Weinheim (2014)

Morini, G.L.: Viscous heating in liquid flows in micro-channels. Int. J. Heat Mass Transf. 48(17), 3637-3647 (2005)

Mottet, L., Coquard, T., Prat, M.: Three dimensional heat and mass transfer in capillary evaporator. In: Proceedings of The 5th International Conference on Porous Media and Their Applications in Science, Engineering and Industry, Kona, Hawaii (2014)

Mottet, L., Coquard, T., Prat, M.: Three dimensional liquid and vapour distribution in the wick of capillary evaporators. Int. J. Heat Mass Transf. 83, 636-651 (2015)

Nishikawara, M., Nagano, H., Mottet, L., Prat, M.: Formation of unsaturated regions in the porous wick of a capillary evaporator. Int. J. Heat Mass Transf. 89, 588-595 (2015) 
Prat, M.: Pore network models for the study of transfer in the porous wick of loop heat pipe. Int. J. Heat Pipe Sci. Technol. 1(2), 129-149 (2010)

Ren, C., Wu, Q., Hu, M.: Heat transfer with flow and evaporation in loop heat pipe's wick at low or moderate heat fluxes. Int. J. Heat Mass Transf. 50(11-12), 2296-2308 (2007)

Ren, C.: Parametric effects on heat transfer in loop heat pipe's wick. Int. J. Heat Mass Transf. 54(17-18), 3987-3999 (2011)

Siedel, B., Sartre, V., Lefèvre, F.: Literature review: steady-state modelling of loop heat pipes. Appl. Therm. Eng. 75, 709-723 (2015)

Surasani, V.K., Metzger, T., Tsotsas, E.: Influence of heating mode on drying behavior of capillary porous media: pore scale modeling. Chem. Eng. Sci. 63(21), 5218-5228 (2008)

Tsotsas, E., Martin, H.: Thermal conductivity of packed beds: a review. Chem. Eng. Process. 22(1), 19-37 (1987)

Wagner, W., Pruß, A.: The IAPWS Formulation 1995 for the thermodynamic properties of ordinary water substance for general and scientific use. J. Phys. Chem. Ref. Data 31(2), 387-535 (2002)

Zhang, X., Li, X., Wang, S.: Three-dimensional simulation on heat transfer in the flat evaporator of miniature loop heat pipe. Int. J. Therm. Sci. 54, 188-198 (2012)

Zhao, T.S., Liao, Q.: On capillary-driven flow and phase-change heat transfer in a porous structure heated by a finned surface: measurements and modeling. Int. J. Heat Mass Transf. 43(7), 1141-1155 (2000) 